Trauma Berufskrankh 2009 • 11[Suppl 1]:85-90 DOI 10.1007/s10039-008-1409-6

Online publiziert: 7. Juni 2008

(c) Springer Medizin Verlag 2008

\section{J.V. Wening}

Abteilung für Unfall-, Hand- und Wiederherstellungschirurgie, Asklepios-Klinik Altona, Hamburg

\title{
Diagnostik und Therapie des Schultertraumas
}

\section{Einfach? Schwierig? Fehlerhaft?}

Traumatische Knochen- und Weichteilverletzungen des Schultergürtels gehören zu den häufigsten Folgen von Verkehrs-, Freizeit- und Sportunfällen.

Das Wort "Schulter" stand im Mittelhochdeutschen für „Grabschaufel", und es gibt zahlreiche Belege, dass unsere Urahnen mit dem Schulterblatt die Gräber für ihre Verstorbenen ausgehoben haben. Kein anderes Gelenk ist in den deutschen Sprachgebrauch so integriert und hat mit Formulierungen wie "Jemandem die kalte Schulter zeigen“, "auf die leichte Schulter nehmen" oder "sich selbst auf die Schulter klopfen" einen breiteren Anklang gefunden. Über 1000 Publikationen pro Jahr, die sich mit Verletzungen (- Tab.1) oder chronischen Veränderungen im Bereich der Schulter beschäftigen, belegen das allgemeine hohe Interesse und lassen einen erheblichen Informationsstand für das Schultergelenk und seine Umgebung erwarten.

Sehr unterschiedliche Ausprägungen eines Schultertraumas verlangen entsprechend unterschiedliche diagnostische Verfahren. Allen gemeinsam in der Diagnostik sind die ausführliche Anamnese und die eingehende klinische Untersuchung. Für die Auswahl der anschließend durchzuführenden Diagnoseverfahren sind Kenntnisse der zu erwartenden Krankheitsbilder unverzichtbar.

\section{Bildgebende Verfahren}

Bei Mehrfachverletzten oder intubierten Schwerverletzten ist inzwischen das so genannte Trauma-Scan „vom Scheitel bis zur Sohle“ allgemein akzeptiert. Anhand des CT (Computertomogramm) können nicht nur zwei-, sondern auch dreidimensionale Schnitte aller gewünschten knöchernen Organe durchgeführt werden, sodass in der Primärdiagnostik das Nativröntgenbild erheblich an Stellenwert verloren hat. Völlig anders sind die Anforderungen an die Diagnostik im Bereich der Poliklinik oder in den Beratungssprechstunden.

Prinzipiell lassen sich 6 Kategorien differenzieren. Abgeklärt/ausgeschlossen werden müssen:

- Komplex der Frakturen aller an der Schulterbildung beteiligten Knochen

- Gefäß-, Weichteil-, Sehnen-, Bänderund Kapselverletzungen

- Tumoren

- Nervenschäden

- Lungen (Pneumothorax)

- Rippen (Frakturen)

Als bildgebende Verfahren stehen für die Weichteile die Sonographie, das MRT (Magnetresonanztomogramm) und die Arthroskopie zur Verfügung. Für den knöchernen Anteil am Schultergelenk werden im Allgemeinen das Nativröntgenbild und das CT, für die Gefäße die Angiographie (Angio-CT) und für besondere Fragestellungen die Szintigraphie verwendet.

In unserer Notfallambulanz mit jährlich etwa 18.000 Patienten werden etwa $3 \%$ mit Schulterfragestellungen vorstellig.

Die anfänglich vorhandene Euphorie über die komplette Digitalisierung sämtlicher, bildgebender Verfahren hat einer gewissen Ernüchterung Platz gemacht. Auch wenn inzwischen das lästige Suchen von Röntgentüten entfällt, verbleiben doch auch in der elektronischen Datenverarbeitung zahlreiche Fehlerquellen, die sowohl den zeitlichen als auch den organisatorischen Ablauf erheblich beeinflussen können. Hauptproblem ist die zwingend notwendige, hohe Auflösung sämtlicher Bildschirme in allen Arbeitsbereichen, um Fissuren zu erkennen oder Überlagerungen korrekt zu interpretieren.

\section{Knöcherne Verletzungen}

\section{Diagnose}

Unverändert trotz aller Neuerungen ist nach einem stumpfen Trauma die bildgebende, primäre Diagnostik zum Ausschluss einer Fraktur in Form einer Nativröntgenaufnahme. Wie bei allen anderen Fragestellungen bezüglich einer knöchernen Gelenkverletzung ist ein Rönt-

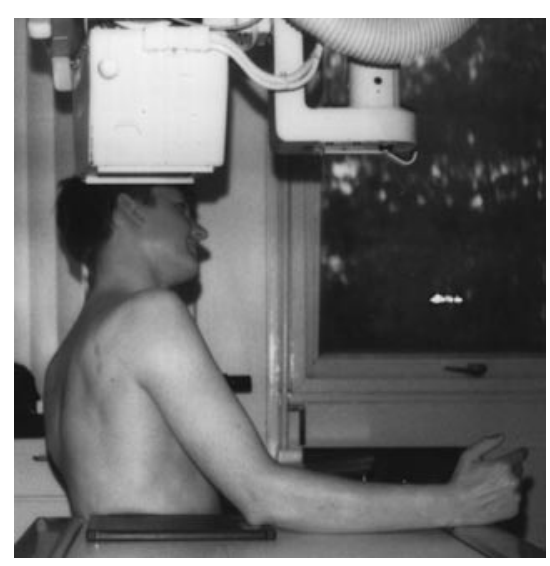

Abb. 1 A Armhaltung bei „indischer Projektion“ 


\section{Der vermeintlich einfache Fall}

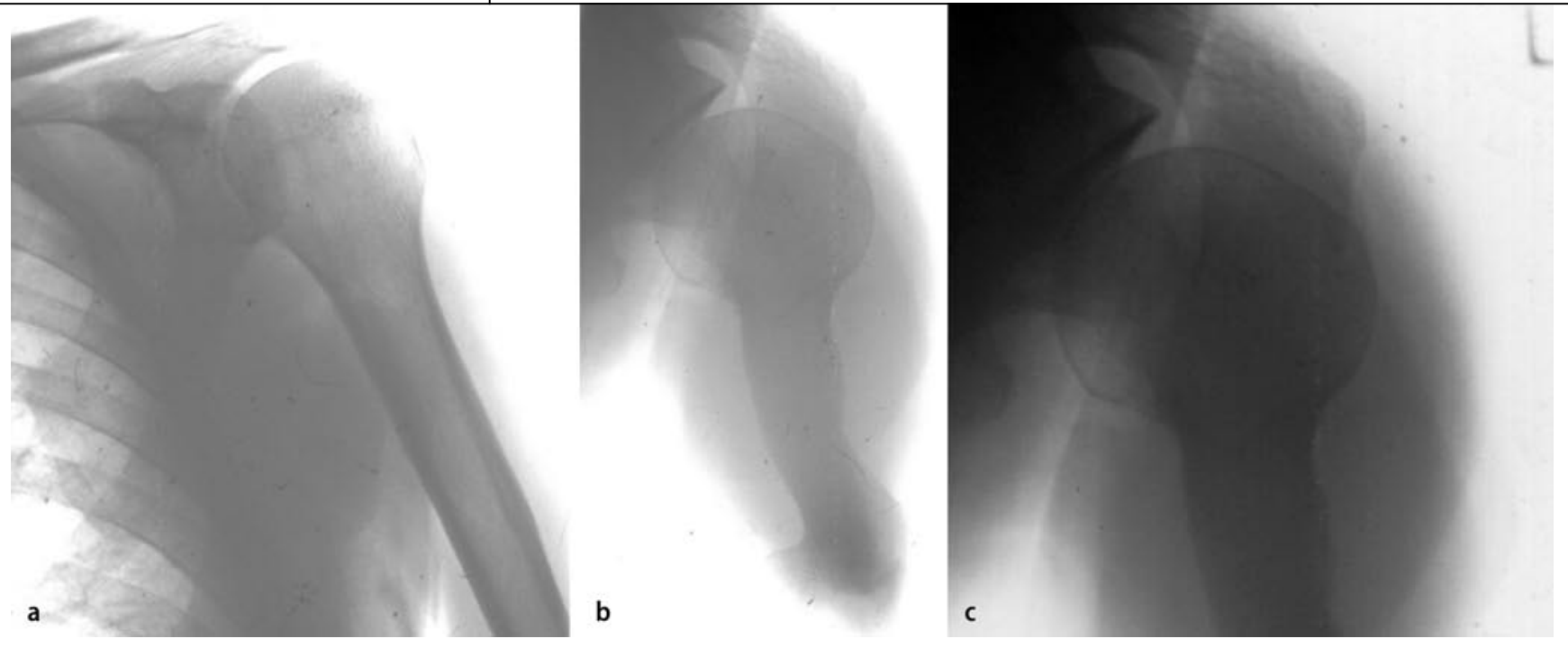

Abb. $2 \Delta$ Verkürzter Humeruskopf mit Pfannendarstellung bei „indischer Projektion“, a a.p. Normalbefund, b, c verformter Humeruskopf durch Projektion

genpaar in 2 senkrecht aufeinander steckenden Ebenen Standard. Diese Anforderung lässt bei der im Raum dreidimensional beweglichen Schulter mehrere Kombinationen zu, von denen sich das a.-p. Röntgenbild und die axiale Röntgenaufnahme als Schultergrundpaar bewährt haben. Die so genannte transthorakale Aufnahme als 2. Ebene ist sehr häufig nicht korrekt zu interpretieren, da es zu zahlreichen Überlagerungen mit der Lunge und an der Schulterbildung beteiligten Knochen kommt. Ergänzend werden Innen- und Außenrotationsaufnahmen durchgeführt. Bei diesen wird die gesunde Schulter zwischen 20 und $45^{\circ}$ nach vorn gedreht, um Kopf und Pfanne wieder überschneidungsfrei mit randständiger, gut ausgeleuchteter Zeichnung des Tuberculum majus darzustellen. Diese Technik ist von der Schulterkopfaufnahme mit flacher Lagerung des Rückens an die Röntgenplatte und Zentralstrahlrichtung auf den Schulterkopf streng zu unterscheiden. Nur in der oben genannten Technik kann man auf dem a.-p. Röntgenbild allein bereits eine hintere Schulterverrenkung sicher nachweisen. Eine andere Ebene als 2. Standardaufnahme ist die Skapulatangentialaufnahme („lateral view“, Skapula-Y) mit einem Aufdrehwinkel von $60^{\circ}$, bei dem der Zentralstrahl parallel zur skapulären Ebene senkrecht auf die Schultergelenkpfanne ausgerichtet ist. Für die Darstellung von Korakoid und Akromion wird die „Cavitasen-Face-Aufnahme“ mit einem Aufdreh- winkel von $45^{\circ}$ verwendet, in welcher sich Korakoid und Akromion wie die kräftigen Arme einer Y-förmigen Zwille darstellen. Die beiden Äste und der Schulterblattkörper formen den unteren Teil des Y.

Die korrekt durchgeführte a.-p. Aufnahme kann selbst bei der hinteren Schulterluxation richtungweisend sein. Auffällig für die hintere Luxation sind die klassischen 6 Befunde:

1. Birnenform des Kopfes $\mathrm{ab} 60^{\circ}$ Innenrotation

2. „rim-sign“ nach Arndt u. Sears [1]: Auf diesen Bildern ist die Distanz zwischen Kopfrand und vorderem Gelenkpfannenrand größer als 6 mm. 3. "trough-line" nach Cisternino et al. [9]: Die ventromedial gelegene Impression des Kopfes stellt sich durch Innenrotation tangential getroffen als kopfrandnahe Spongiosaverdichtung dar.

4. Moloney's Linie nach Dorgan [11]: Es besteht eine Unterbrechung des gotisch anmutenden Skapula-HumeralBogens im tangentialen Röntgenbild.

5. Fehlender Halbmond nach Nobel [26]: Überlappung von Kopf und Pfanne mit leerem unterem Pfannenanteil

6. Kopfhochstand

Der Nachweis eines Bruchs des Processus coracoideus bei basisnahen Frakturen gelingt am besten bei abduziertem oder angehobenem Arm mit $45-60^{\circ}$ Einfallswinkel.
Einen geringen Ausschnitt des Sulcus bicipitalis ermöglicht die Tangentialaufnahme nach Fisk [13]. Strahlungsfrei ist die sonographische Untersuchung, wobei sich der Sulcus in beiden Ebenen mit hoch auflösenden Schallköpfen (7,5/10 MHz) exzellent darstellen lässt.

\section{Verlaufskontrolle}

Das Ergebnis chirurgischer Bemühungen postoperativ oder nach Reposition des Schultergelenks in einem ruhig stellenden Verband oder einem Gips zu kontrollieren, gehört zu den standardisierten Vorgehensweisen. In Anlehnung an die Pendelaufnahmen von Corradi u. Del Moro [10] im ventrodorsalen Strahlengang von $45^{\circ}$ kranial nach kaudal und im Weiteren dem ,angle-up-view“ von Bloom u. Obata [6] ist die „axillary view“ nach Velpeau sinnvoll: Der am Röntgentisch lehnende Verletzte beugt sich etwa $30^{\circ}$ nach hinten, sodass der Zentralstrahl senkrecht durch die Schulter von kranial nach kaudal geführt wird. Diese Lagerung ist nicht mit Schmerzen verbunden und kann auch bei älteren Patienten durchgeführt werden. Eine ähnliche Projektion ist die Aufnahme nach Mukherjee-Syvaya [25] (indische Projektion). Hierbei wird von kranial nach kaudal ohne Abduktion, Elevation oder Retroversion bei etwa $20^{\circ}$ nach vorn gewinkeltem Arm geröntgt (• Abb. 1, 2). In dieser Projektion wird der Oberarmkopf in Relation zur Pfanne vergrößert dargestellt, und man kann sowohl vordere als auch hintere Impres- 
sionen oder eingekeilte Luxationen ohne Zweifel erkennen.

\section{Verfahrenswahl}

Zur Festlegung des therapeutischen Konzepts bei Schulterluxationen hat sich die Bildgebung eindeutig in Richtung des CT bzw. für die Limbusbeurteilung in Richtung des MRT verschoben. Aufnahmen wie der Westpoint-View, der zur vorderen unteren Pfannenranddiagnostik empfohlen wurde, sind weitgehend durch die Computertomographie abgelöst worden. Auch für unklare Befunde im Skapulabereich wird das CT herangezogen, wobei es exakt zur Ausmessung des glenohumeralen Winkels ohne zusätzliche Aufnahmen verwendet werden kann. In computertomographischen Aufnahmen werden die traumatischen Humeruskopfveränderungen wie der Hill-Sachs-Defekt ventral und dorsal klar und eindeutig nachgewiesen. Mehrfragmentfrakturen sind zur differenzierten Therapie als Entscheidungshilfe für einen Rekonstruktionsversuch oder eine Schulterprothese sicher ebenfalls am besten mit dem CT dargestellt.

Aus einer Vielzahl von Möglichkeiten sind somit zahlreiche, radiologische Abklärungsvorschläge für die Folgen einer Schulterluxation definiert. Empfohlen werden sollten, wenn kein CT durchgeführt wird:

1. Schulter a.-p. (Schulterkopfaufnahme in $60^{\circ}$ Innenrotation)

2. Schulter axial

3. Pfannenprofilaufnahme nach Bergano [3]

4. Dorsale Tangentialaufnahme in der Technik von Saxer [30] und Johner [19] oder Hermodsson [16]

Operativ therapeutisch sind die Ergebnisse bei subkapitalen Frakturen und $\mathrm{Hu}$ meruskopffrakturen, die rekonstruktionsfähig sind, bei Verwendung von winkelstabilen Platten und Nägeln gleich.

\section{Weichteilverletzungen}

Bei allem Augenmerk, das sich nach einem Schultertrauma auf den Knochen richtet, müssen die Nerven-, Gefäß- und Sehnenverletzungen bei entsprechenden
Tab. 1 Mögliche Frakturen an der

Schulter

Subkapitale Humerusfraktur

Humeruskopffraktur

Skapulafraktur

Korakoidfraktur

Akromionfraktur

Humeruskopfnekrosen

Klavikulafraktur

Akromioklavikularsprengung

Tab. 2 Zoneneinteilung der Rotatorenmanschette

\section{Zone Beschreibung}

A Subskapularissehne und lange Bizepssehne

B Supraspinatussehne

C Infraspinatus- und Teres-minorSehne

Tab. 3 Einteilung der inkompletten Rotatorenrupturen. (Nach [11])

Größe $($ Breite $[\mathrm{mm}] \times$ maximale Retention [mm])

\begin{tabular}{ll}
\hline Grad 1 & $<3 \mathrm{~mm}$ tief \\
\hline Grad 2 & $3-6 \mathrm{~mm}$ tief \\
\hline Grad 3 & $>6 \mathrm{~mm}$ tief \\
\hline Lokalisation & \\
\hline A-Läsion & Artikulär- oder gelenkseitig \\
\hline B-Läsion & Bursa- oder akromionseitig \\
\hline C-Läsion & Intratendinös \\
\hline Ursachen der & Rotatorenmanschettenläsion \\
\hline 1. & Degeneration \\
\hline 2. & Impingement \\
\hline 3. & Stumpfes Schultertrauma \\
\hline 4. & Schulterluxation \\
\hline
\end{tabular}

oder unklaren klinischen Befunden in die Betrachtung einbezogen werden.

\section{Nervenverletzungen}

Bei Nervenschäden mit Paralyse des N. axillaris treten die Symptome in unterschiedlichster Ausprägung auf. Das klinische Bild ist gekennzeichnet von einer Hängeschulter - wie bei der posttraumatischen Pseudoparalyse des M. deltoideus (- Abb. 3). Richtungweisend ist auch eine Sensibilitätsstörung am proximalen, lateralen Oberarm.

Pseudoparalyse und N.-axillaris-Schäden treten in bis zu 30\% der Schulterverletzungen, insbesondere nach vorderen Luxationen, bezogen auf den N. axillaris als Dehnungsschäden auf, die in einem
Trauma Berufskrankh 2009 11

[Suppl 1]:85-90

DOI 10.1007/s10039-008-1409-6

○) Springer Medizin Verlag 2008

\section{J.V. Wening \\ Diagnostik und Therapie des Schultertraumas. Einfach? Schwierig? Fehlerhaft?}

\section{Zusammenfassung}

Beim stumpfen Schultertrauma besteht die nach der klinischen Untersuchung durchgeführte primäre bildgebende Diagnostik aus dem Nativröntgenbild, beginnend in 2 senkrecht zueinander stehenden Ebenen. Zahlreiche Zusatzaufnahmen ermöglichen eine differenzierte Diagnostik der an der Gelenkbildung beteiligten Knochen, unklar verbleibende Befunde werden durch die Computertomographie (CT) geklärt. Die Magnetresonanztomographie (MRT) und die Sonographie sind die klassischen Verfahren für die Weichteildiagnostik. Die Gefäßdiagnostik ist an die konventionelle Angiographie, das Angio-CT oder Angio-MR gebunden.

\section{Schlüsselwörter}

Stumpfes Schultertrauma · Röntgendiagnostik nativ - Computertomographie - Magnetresonanztomographie $\cdot$ Angiographie

\section{Diagnosis and therapy of shoulder trauma. Easy? Difficult? Defective?}

\section{Abstract}

Blunt trauma of the shoulder needs, after clinical investigation, $x$-ray examination to exclude fractures. Despite technical progress, radiographs in two planes are still basic diagnostic tools. Computed tomography (CT) is necessary in cases of differential questions for fracture treatment. Magnetic resonance imaging (MRI) or ultrasound is the method of choice in cases of rotator cuff tears and soft tissue injuries. Vascular diseases and injuries can be diagnosed by the use of conventional angiograms or angio-CT and angio-MRI.

\section{Keywords}

Blunt shoulder trauma . Conventional x-ray .

Computed tomography . Magnetic resonance imaging $\cdot$ Angiography 


\section{Der vermeintlich einfache Fall}

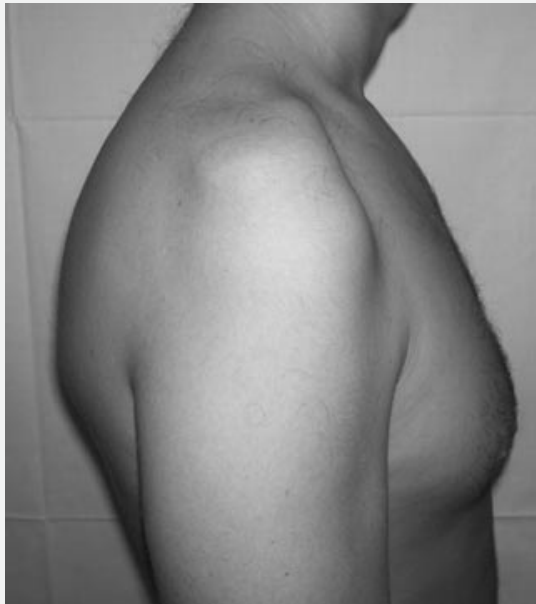

Abb. $3 \Delta$ Atrophie des M. deltoideus bei neurogener Schädigung

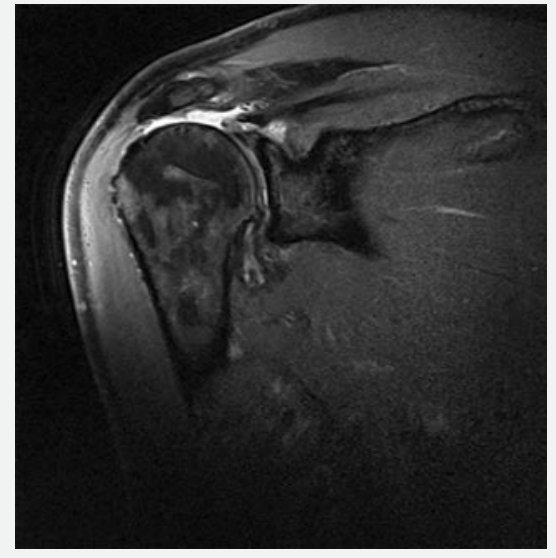

Abb. 4 \ MRT: Gelenkerguss, Ruptur der Supraspinatussehne mit Retraktion

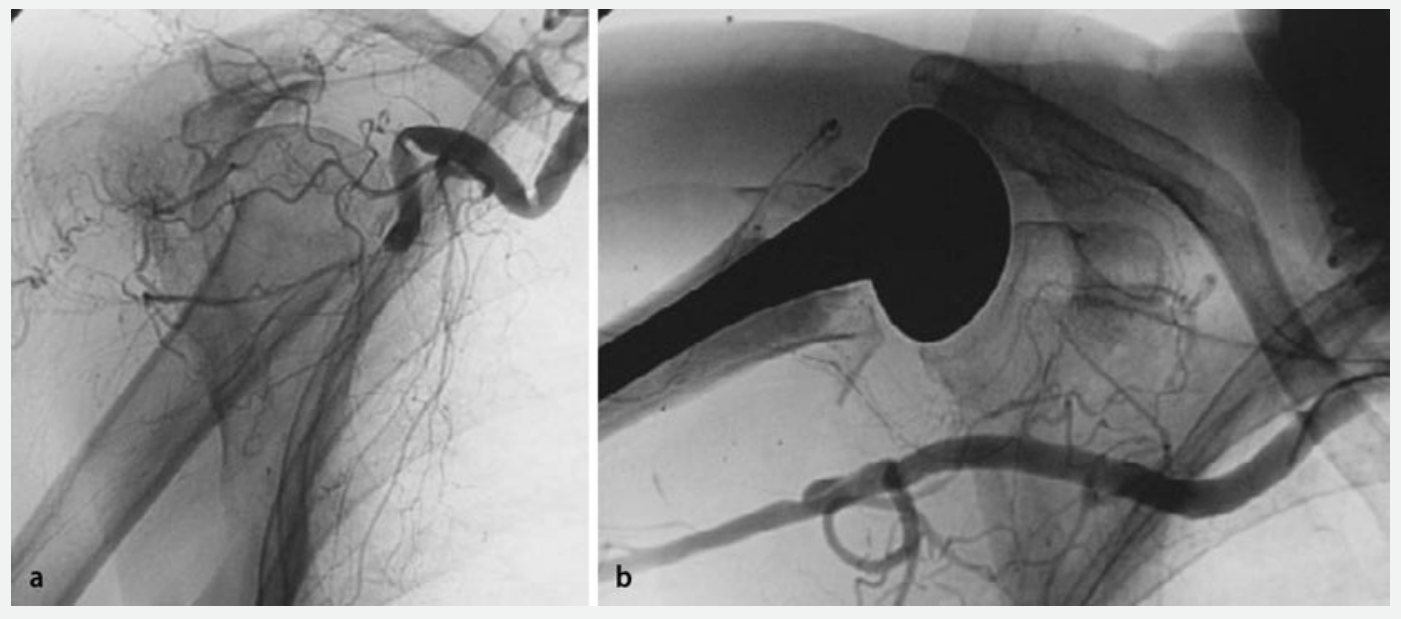

Abb. $5 \varangle$ Humeruskopfluxationsfraktur mit Abklemmung der $A$. axillaris (a), freier Durchfluss nach operativer Versorgung mit Reposition und endoprothetischem Ersatz (b)

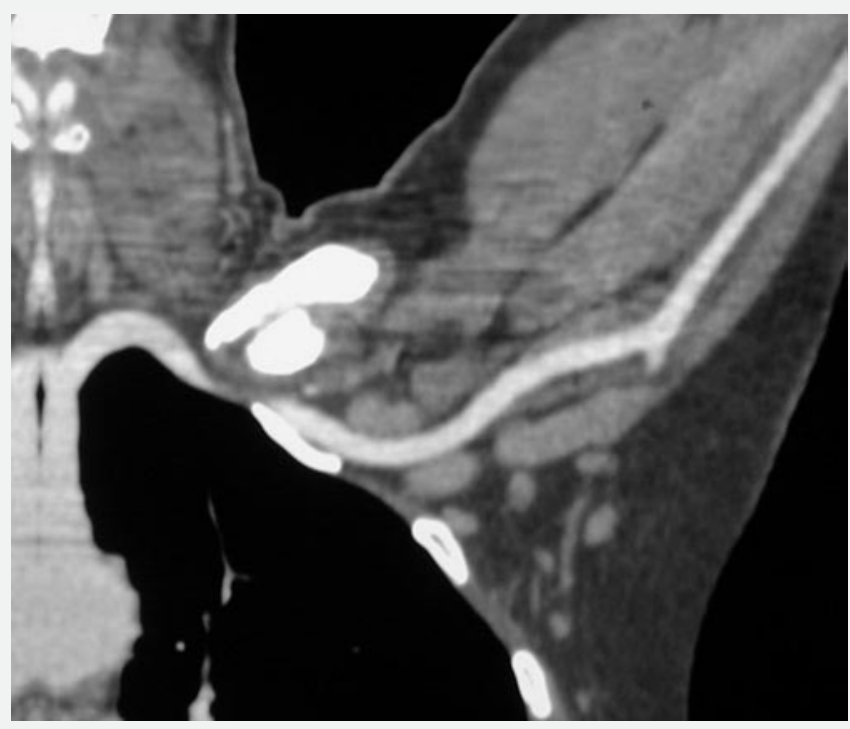

Abb. $6<$ Schulter-Angio-CT bei Kompression $\operatorname{der} \mathrm{A}$. axillaris durch vermehrte Kallusbildung nach Klavikulafraktur 


\begin{tabular}{|c|c|}
\hline Grad & Beschreibung \\
\hline 0 & $\begin{array}{l}\text { Normale Muskulatur ohne Fett- } \\
\text { streifen }\end{array}$ \\
\hline I & Muskulatur mit einigen Fettstreifen \\
\hline ॥ & $\begin{array}{l}\text { Deutliche fettige Infiltration, aber } \\
\text { mehr Muskulatur als Fett }\end{array}$ \\
\hline III & $\begin{array}{l}\text { Fettige Degeneration mit gleich viel } \\
\text { Fett wie Muskulatur }\end{array}$ \\
\hline IV & $\begin{array}{l}\text { Fettige Degeneration mit mehr Fett } \\
\text { als Muskulatur }\end{array}$ \\
\hline
\end{tabular}

hohen Prozentsatz reversibel sind und sich innerhalb von 3 Monaten bis $1 \mathrm{Jahr}$ zurückbilden. Hilfreich in der Beurteilung ist das EMG (Elektromyogramm). Bei Schädigung des $\mathrm{N}$. axillaris wird nach 3-6 Monaten eine Kontrolle empfohlen. Besteht ein vollständiger Ausfall, wird von einem vorderen und hinteren $\mathrm{Zu}$ gang aus ein Nerveninterponat (N. suralis) notwendig, wobei in der Literatur die Ergebnisse als relativ günstig beschrieben werden, je früher der Eingriff vorgenommen wird. Das Resultat wird bei verzögerter Behandlung durch bereits erfolgte Hypotrophie der versorgten Muskulatur negativ beeinflusst.

\section{Syndrom des Spatium quadrilaterale (axillares Engpasssyndrom)}

Das Syndrom sollte als Rarität ohne Trauma bekannt sein. Bei ihm geben wenig spezifische Beschwerden wie diffuser Schulterschmerz und Parästhesien im Arm zu häufigen Arztkonsultationen Anlass. Der objektivierbare Nachweis ist ein Kompressionsverschluss der dorsalen A. circumflexa humeri, sichtbar in der Angiographie über die A. subclavia.

Therapeutisch hilft nur die operative Dekompression.

\section{Rotatorenverletzungen}

Für die Diagnostik der Rotatorenruptur wird als Diagnostikum der ersten Wahl die Sonographie empfohlen, die in über 90\% richtungweisende Befunde ergibt. Unabhängig davon ist die MRT für die Weichteilbeurteilung der Schulter der so genannte Goldstandard, wobei ihr Ergebnis nicht nur durch die verwendete Technik, sondern auch durch die Fragestellung beeinflusst wird. Wenn die Schul-

\begin{tabular}{|c|c|c|c|}
\hline Grad & $\begin{array}{l}\text { Typische } \\
\text { Größe }[\mathrm{cm}]\end{array}$ & Bezeichnung & Beschreibung \\
\hline 0 & - & Normale Sehne & $\begin{array}{l}\text { Intakte Rotatorenmanschette mit glatter Bedeckung } \\
\text { durch Synovia und Bursa }\end{array}$ \\
\hline 1 & $<1$ & Minimale Läsion & $\begin{array}{l}\text { Oberflächliche bursale oder synoviale Irritation oder } \\
\text { leichte Ausfransung der Kapsel in einem kleinen, um- } \\
\text { schriebenen Bezirk }\end{array}$ \\
\hline 2 & $<2$ & Echter Teilschaden & $\begin{array}{l}\text { Ausfransungen oder Versagen einiger Sehnenfasern; } \\
\text { zusätzlich synoviale, bursale oder kapsuläre Läsion }\end{array}$ \\
\hline 3 & $<3$ & $\begin{array}{l}\text { Ausgeprägter Teil- } \\
\text { schaden }\end{array}$ & $\begin{array}{l}\text { Fransen- und Rissbildung in Sehnenfasern; oft die } \\
\text { gesamte Oberfläche einer Sehne betreffend, meist der } \\
\text { Supraspinatussehne }\end{array}$ \\
\hline 4 & $>3$ & $\begin{array}{l}\text { Sehr schwerer Teil- } \\
\text { schaden }\end{array}$ & $\begin{array}{l}\text { Zusätzlich zur Fransen- und Rissbildung in Sehnenfa- } \\
\text { sern meist Lappenriss einer Sehne sowie Beteiligung } \\
\text { mehr als einer Sehne; Übergang in kompletten Defekt }\end{array}$ \\
\hline
\end{tabular}

ter im MRT nach einem Trauma beurteilt werden soll, müsste auch eine Befundung der Bizepssehne, des Limbus und des Intervalls vorgenommen werden.

Bei der Beschreibung von Rotatorenverletzungen ist die Diktion in vielen schriftlichen Befunden trotz vorhandener Klassifikationen ( $\bullet$ Tab. 2, 3, 4, 5) erfahrungsgemäß unscharf.

Die Rissform kann vielseitig und vielschichtig sein und zahlreiche Varianten aufweisen wie eine komplette Ruptur der Supraspinatussehne (- Abb. 4), eine inkomplette Querruptur, eine Ruptur im Faserverlauf, eine Beteiligung der Subskapularissehne, der Infraspinatussehne und des M. teres minor oder auch Kombinationen.

Liegt eine fettige Degeneration der Muskulatur vor, ist davon auszugehen, dass sich diese nicht zurückbildet - auch nicht nach operativer Rekonstruktion.

\section{SLAP-Läsion („,superior labrum anterior to posterior lesion")}

Konkurrenzlos etabliert hat sich die MRTDiagnostik unter den bildgebenden Verfahren für die Verletzungen des Labrum glenoidale am oberen Rand der Schulterpfanne (SLAP-Läsionen, häufig bei Sportlern, die sich bei Überkopfsportarten verletzen) sowie für die Limbusbeurteilung nach Schulterluxationen. Eine Kontrastmittelgabe im MRT ist obligatorisch für eine komplette Befundung der Weichteile.

Operativ-therapeutisch ist bei diesen Verletzungen die arthroskopisch kontrollierte Refixation der genannten Struktu- ren durch Anker häufig die Behandlung der Wahl.

\section{Operative Maßnahmen}

Sie sind sowohl arthroskopisch als auch als offener Eingriff (Mini-open-Repair-Technik) möglich. Kleinere Risse und Verletzungen sind in vielen Zentren und Spezialkliniken eine Domäne der arthroskopischen Chirurgie; Massenrupturen werden eher in offener Verfahrensweise refixiert. Bezüglich der „einfachen“ Rotatorenmanschettenrupturen halten sich die Empfehlungen in etwa die Waage.

Akzeptiert sind sowohl die Mini-openRepair-Technik als auch die arthroskopische Refixation. Fortschritte sind durch modifizierte Instrumente und so genannte Anker in verschiedensten Variationen erreicht worden. Ob die Refixation der Supraspinatussehne einreihig oder zweireihig erfolgen sollte, hängt sicher auch mit der noch vorhandenen Qualität der Sehnen zusammen. Ein Anfrischen des Knochens am Ansatzpunkt wird als Voraussetzung für eine Heilung angesehen. Bei schweren degenerativen Veränderungen (fettige Degenerationen) sind die Ergebnisse erfahrungsgemäß schlechter. Die von Versicherungen gerne in Anspruch genommene Klassifizierung der Rotatorendegeneration unter Berücksichtigung intraoperativ entnommener Histologien trägt für den Gutachter manchmal eher zur Verwirrung als zur Klärung der Situation bei. 


\section{Gefäßverletzungen}

Sie sind nicht nur Folge eines akuten Traumas (Gefäßkompression in der Axilla durch Blutungshämatom nach Schulterluxation, - Abb.5), sondern können auch im Rahmen von überschießender Knochenneubildung (Kallushypertrophie nach Klavikulafraktur) durch zunehmende Kompression entstehen (- Abb.6). Auch die subkapitale Humerusfraktur ist durchaus in der Lage, eine Durchblutungsstörung auszulösen.

\section{Fazit für die Praxis}

Nach einem Schultertrauma sind die Anamnese und der klinische Befund trotz zahlreicher, apparativer, technischer Untersuchungsmöglichkeiten die Basis des ärztlichen Handelns. Die Kenntnisse der verschiedenen Krankheitsbilder sind Voraussetzung, um z. T. kostenintensive, bildgebende Verfahren im weiteren Verlauf zu rechtfertigen oder zu vermeiden. In den meisten Fällen sind einfache Röntgenaufnahmen in 2 Ebenen Ausgangspunkt für eine erste Diagnose. Spezialaufnahmen erhärten dieselbe und ergänzen das diagnostische Spektrum. Unverändert sind für eine weiterführende Knochendiagnostik auch im Gelenkbereich das $C T$, für Weichteilbefunde die Sonographie alternativ oder summativ zum MRT das Diagnostikum der Wahl bei differenzierten Fragestellungen.

\section{Korrespondenzadresse}

\section{Dr.J.V.Wening}

Abteilung für Unfall-,

Hand- und Wiederherstellungschirurgie,

Asklepios-Klinik Altona

Paul-Ehrlich-Straße 1, 22763 Hamburg

j.wening@asklepios.com

Interessenkonflikt. Keine Angaben.

\section{Literatur}

1. Arndt JH, Sears AD (1965) Posterior dislocation of the shoulder. Am J Roentgenol Radium Ther Nucl Med 94: 639-645

2. Bateman JR (1963) The diagnosis and treatment of ruptures of the rotator cuff. Surg Clin North Am 43: 1523-1530

3. Bernageau J, Potte D, Debeyre J et al. (1976) Intérêt du profil glénoidien dans les luxations récidivantes de l'epaule. Rev Chir Orthop Reparatrice Appar Mot [Suppl 2] 62: 142-147
4. Bernau A (1982) Orthopädische Röntgendiagnostik. Einstelltechnik. Urban \& Schwarzenberg, München Wien Baltimore

5. Bitzer M, Nasko M, Krackhardt T et al. (2004) Direkte CT-Arthrographie versus direkte MR-Arthrographie bei chronischer Schulterinstabilität: Ein Methodenvergleich nach Einführung der Multidetektor-CT-Technik. Röfo 176: 1770-1775

6. Bloom MH, Obata WG (1967) Diagnosis of posterior dislocation of the shoulder with use of Velpeau axillary and angle-up roentgenographic views. J Bone Joint Surg Am 49: 943-949

7. Bureau NJ, Beauchamp M, Cardinal E et al. (2006) Dynamic sonography evaluation of shoulder impingement syndrome. AJR Am J Roentgenol 187: 216-220

8. Busquets AR, Acosta JA, Colon E et al. (2004) Helical computed tomographic angiography for the diagnosis of traumatic arterial injuries of the extremities. JTrauma 56: 625-628

9. Cisternino SJ, Rogers LF, Bradley CS et al. (1978) The through line: a radiographic sign of posterior shoulder dislocation. AJR Am J Roentgenol 130: 951

10. Corradi C, Del Moro VM (1953) La lussazione posteriore della spalla contributo radiologico. Arch Orthop 66: 475

11. Dorgan JA (1955) Posterior dislocation of the shoulder. Am J Surg 89: 890-900

12. Ellmann H (1990) Diagnosis and treatment of incomplete rotator cuff tears. Clin Orthop 254: 6474

13. Fisk C (1965) Adaption of the technique for radiography of the bicipital groove. Radiol Techn 37: 47

14. Froimson Al (1978) Fracture of the coracoids process of the scapula. J Bone Joint Surg Am 53: 710

15. Goutallier D, Postel JM, Bernageau J et al. (1994) Fatty muscle degeneration in cuff ruptures - preand post-operative evaluation by CT-scan. Acta Orthop Scand 304: 78-83

16. Hermodsson I (1934) Röntgenologische Studien über die traumatischen und habituellen Schultergelenksverrenkungen nach vorn und nach unten. Acta Radiol [Suppl 20] 14: 1

17. Hill HA, Sachs MD (1940) The grooved defect of the humeral head. Radiology 35: 690

18. Jain S, Monbaliu D, Thompson JF (2002) Thoracic outlet syndrome caused by chronic retrosternal dislocation of the clavicle. Successful treatment by transaxillary resection of the first rib. J Bone Joint Surg Br 84: 116-118

19. Johner R, Burg HB (1984) Radiologische Diagnostik bei Schulterluxation. In: Chapchal G (Hrsg) Verletzungen und Erkrankungen der Schulterregion. Thieme, Stuttgart New York

20. Kraft CN, Fahmy U, Nicolay C et al. (2004) Wertigkeit der Sonographie nach Rotatorenmanschettennaht: ein Vergleich mit MRT und klinischer Diagnostik. Ultraschall Med 25: 40-47

21. Kwon YW, Powell KA, Yum JK et al. (2005) Use of three-dimensional computed tomography for the analysis of the glenoid anatomy. J Shoulder Elbow Surg 14: 85-90

22. Lawrence WS (1918) A method of obtaining an accurate lateral roentgenogram of the shoulder joint. AJR Am J Roentgenol 5: 193

23. Lester B, Jeong GK, Weiland AJ et al. (1999) Quadrilateral space syndrom: diagnosis, pathology, and treatment. Am J Orthop 28: 718-725

24. Melloni G, Giovanardi M, De Gaspari A et al. (2006) Transient thoracic duct obstruction in a patient with thoracic outlet syndrome. Eur J Cardiothorac Surg 30: 674

25. Mukherjee-Syvaya (persönl. Mitteilung)
26. Nobel W (1962) Posterior traumatic dislocation of the shoulder. J Bone Joint Surg (Am) 44: 523

27. Ozcakar L, Kaymak B, Turan S et al. (2006) Thoracic outlet syndrome, Paget-Schroetter syndrome and aberrant subclavian artery in a young man. Joint Bone Spine 73: 469-471

28. Ozcan F, Ozcakar L, Ozcan HN et al. (2006) Thoracic outlet syndrome masquerading as a diagnostic pitfall in Behcet's disease. Rheumatol Int 26: 865866

29. Pennekamp W, Gekle C, Nicolas V et al. (2006) Initiale Ergebnisse der Schulter-MRT in Außenrotation bei primärer Schulterluxation und nach Ruhigstellung in Außenrotation. Röfo 178: 410-415

30. Saxer U (1978) Indikation und Technik der Limbusverschraubung nach M.E. Müller nach habitueller Schulterluxation. Orthopaede 7: 160

31. Schlecht I, Gaffke G, Stroszczynski C et al. (2001) Diagnostik von Tumoren des Schultergürtelbereichs in der MRT. Röntgenpraxis 53: 191-195

32. Schroder RF, Bostanjoglo M, Kaab M et al. (2003) Treffsicherheit der nativen und kontrastverstärkten MRT im Routineeinsatz bei Supraspinatussehnenrupturen - Vergleich mit operativen Ergebnissen. Röfo 175: 920-928

33. Snyder SJ, Pachelli AF, Del Pizzo W et al. (1991) Partial thickness rotator cuff tears - results of arthroscopic treatment. Arthroscopy 7: 1-7

34. Vanderstraeten G, Ozcakar L, Verstraete K (2006) Thoracic outlet syndrome portending Klippel-Feil syndrome. Joint Bone Spine 73: 763-764

35. Waldt S, Metz S, Burkart A et al. (2006) Variants of the superior labrum and labro-bicipital complex: a comparative study of shoulder specimens using MR arthrography, multi-slice CT arthrography and anatomical dissection. Eur Radiol 16: 451-458

36. Wulker N, Ruhmann O (2001) MRT bei Luxationen und Instabilität des Schultergelenks. Orthopäde 30: 492-501 